\section{Introduction to Materials for Advanced} Energy Systems

Introduction to

Materials for

Advanced Energy

Systems

\section{Colin Tong}

Springer, 2019

911 pages, $\$ 119$

ISBN 978-3-319-98002-7 (eBook)

\section{을 Spring}

$\mathrm{T}$

his textbook reviews various materials used in all types of energy systems - both traditional and advanced. Energy systems cover a wide range, from energy sources, to conversion methods, and to storage options. Renewables and sustainable energy systems are the current focus of developmental activities.

The book addresses energy and the environment from a materials science and engineering point of view. It is divided into 12 chapters. The first chapter is a general introduction to all types of energy systems - not only advanced energy systems, but also well-established ones. The second chapter is a cursory introduction to the science and engineering of different materials used in energy systems. It also highlights materials characterization and testing techniques.

The following chapters deal with materials used in various energy systems, such as fossil, solar, geothermal, and wind. Chapter 3 covers materials used in fossil fuel energy systems, including how materials play an important role in improving efficiency and reducing greenhouse gas emissions. Chapter 4 discusses solar-energy materials (e.g., photovoltaic, thermal collectors, solar absorbers), and Chapter 5 describes materials used in the geothermal industry, including pumps, heat exchangers, drill bits, and cementing of wells. Chapter 6 focuses on wind energy and materials used in making wind turbines and for energy conversion.

Chapter 7 deals with materials for hydropower and other ocean-based energy systems, such as tidal, wave, ocean current, ocean thermal, and salinity gradient. Chapter 8 focuses on biomass and bioenergy materials, whereas Chapter 9 covers hydrogen as a fuel source and energy-storage medium and various types of fuel-cell technologies. Various materials used as nuclear fuels, fuel cladding, moderators, reflectors, coolants, and structural aspects of current and new fission reactors are covered in Chapter 10. Chapter 11 describes the science behind emerging materials for energy harvesting, such as thermoelectric, piezoelectric, pyroelectric, triboelectric, magnetoelectric, and other hybrid systems. Future trends such as metamaterials, nanomaterials, artificial photosynthesis, and zeropoint energy are described in Chapter 12.

The book succeeds in comprehensive coverage of materials used in energy systems, their current usage, new developments, and future prospects. It covers the core science behind new systems from nano, through micro, to macro systems. Each chapter includes recent references. It is written in simple language but assumes some basic background in materials science. If anyone wants to make an investment decision in the energy sector, he/ she needs to know the future trends in the energy systems. This book highlights such R\&D activities.

Overall, it is a good book for engineers and managers who are involved in any energy industry, renewable or otherwise. The reader should have a good understanding of the science and engineering aspects of materials, starting with quantum physics and electrochemistry, to fully comprehend some of the new developments. This book gives a nice overview of developments in competing energy systems, which is valuable to business executives in the energy industry.

Reviewer: Chinnia Subramanian, Technical Consultant, EnMat Solutions Inc., Canada.

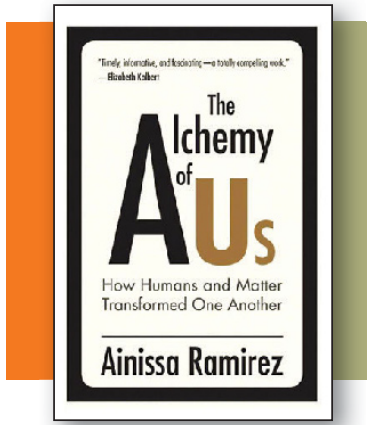

The Alchemy of Us

Ainissa Ramirez

The MIT Press, 2020

328 pages, $\$ 55.00$

ISBN 978-0-262-04380-9

What do Ruth Belville, Albert Einstein, Louis Armstrong, and David Eagleman have in common?

In unpredictable ways, Ainissa Ramirez melds their stories together as she mixes social history with materials science in a fascinating book for the general public. She presents more than a synergistic relationship between science and societal needs. The Alchemy of Us, Ramirez writes, "shows how materials were shaped by inventors, but also how those materials shaped culture." This angle, then, also renders the book interesting for the materials research community.

For example, in the Western world, the invention of artificial light led to a new sleep pattern, which then drew a need for human-made timepieces. That, in turn, altered our construct of time. This is where Ruth Belville comes into the story.

In 1908, Belville made her rounds in London with her trusty pocket watch named Arnold. Because of exquisite developments in materials science accomplished by an inventor born 200 years earlier, the steel spring - along with the brass gears and ruby pivots - clicked five times 
a second. Belville compared Arnold to the master clock at the Royal Observatory in Greenwich, received a certificate identifying the difference, then proceeded to "sell" time to her customers, who used Belville's information to determine the accurate time against their own clocks.

This pursuit of precision in timekeeping continued to elicit materials innovations. One of these was the use of piezoelectricity in quartz that led to incorporating the gem in a clock. "For generations," Ramirez writes, "society has struggled to make better and better clocks so we can coordinate our interactions during the day." Ramirez's study does not stop there, though. With further exploration in physics (Einstein), music (Armstrong), and neuroscience
(Eagleman), she shows how "precision"that holy grail—keeps evading our reach.

With other examples of materials research, Ramirez bridges Abraham Lincoln, a patent race between Sir Henry Bessemer and William Kelly, and the advent of Christmas caroling. She traces the evolution of materials for photography as they affected the progress and regression of social bias in the United States and South Africa. In addition, a recurring theme in the book is how materials change the "shape of data," which subsequently changes the human brain, concerns of which are still under intense research.

What makes the book a fun read is not just the "information," but also the stories Ramirez tells. As a writer, I'm mesmerized by the choices she makes to connect with the general reader, which I'm sure to probe later in the MRS Bulletin blog, Materials Connect.org.

I can see this book making it to the "gift list" for (1) family and friends, so that they can understand the field I work in; (2) science writers, so they can study a grand example of how to tell a story that makes materials research understandable to the general population; and (3) materials researchers, who can see their work from a perspective beyond the laboratory.

Reviewer: Judy Meiksin, News Editor, MRS Bulletin.

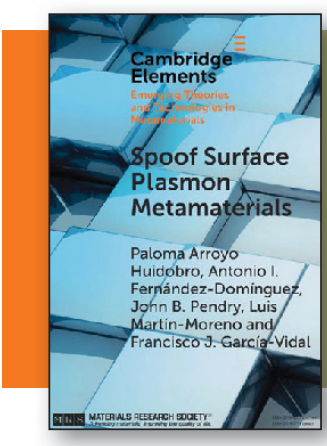

\section{Spoof Surface Plasmon Metamaterials} Paloma Arroyo Huidobro, Antonio I. FernándezDomínguez, John B. Pendry, Luis Martín-Moreno, and Francisco J. García-Vidal Cambridge University Press and Materials Research Society, 2018, 123 pages, \$25 (paperback), ISBN 978-1-108-45105-5

In the interest of transparency, MRS is a copublisher of this title. However, this review was requested and reviewed by an independent Book Review Board.

T his book is a unique part of a series of "emerging theories and technologies in metamaterials." This series provides systematic coverage of new and emerging topics in metamaterials, which is a timely elaboration of metamaterials for scientists, engineers, and students. The presentation of recent and desirable details is given in a logical and systematic manner in six sections. It encompasses the basics and present day technological updates that show how the experimental ideas of metamaterials applications can be realized.

Theoretical concepts and solid-state physics-based details of metamaterials are introduced in Section 1, which highlights the desired details on metamaterials, plasmonics, and spoof surface plasmons (SSPs). An in-depth section on theoretical formalism is given in Section 2. These two sections provide the background necessary for understanding the subject.

Sections 3 and 4 provide a thorough description of SSPs in flat geometries, providing an understanding of waveguide-related SSPs. Section 3 focuses on the basic interpretation of SSPs on various flat configurations viz. flat surfaces (straight/slanted groves/square dimples), perforated slabs, and related SSP metamaterial parameters. Further, it provides information on the preparation of flat SSP metamaterials. The approach discussed in the section enables the reader to interpret various configurations and applications of plasmonic metamaterials.

Section 4 elaborates on the theoretical aspects of SSP waveguides, with a focus on cylindrical geometries and experimental work reported in the literature. It directs the reader to correlate the theory of metamaterials to cases close to the application of SSP metamaterials. It describes cases such as corrugated channel waveguides, wedges, Domino surface plasmons, and conformal surface plasmons. Section 5 is a larger section that explains the localized surface plasmons that can be observed by way of nanostructuring of metallic systems, which leads to experimental realization of any kind of application. The book teaches how metallo-dielectric disks and magnetic localized surface plasmons in pure metallic structures can be exploited for possible applications of metamaterials. The book concludes with Section 6, which summarizes the content.

This book clearly demonstrates the status and importance of metamaterials by covering theoretical and experimental aspects of this new technology. The figures and equations are appropriately chosen and displayed to provide insights into various aspects pertaining to the subject. All recent work has been suitably cited via references in the field. This will be a valuable book to readers who are looking for concise recent information on this new and upcoming field. This would be a useful book to inspire and provide desirable details to scientists and students interested in metamaterials.

Reviewer: Pramod H. Borse, International Advanced Research Centre for Powder Metallurgy and New Materials (ARCI), India. 